\title{
Measurement of $\mathbf{M}^{2}$-Curve for Asymmetric Beams by Self-Referencing Interferometer Wavefront Sensor
}

\author{
Yongzhao $\mathrm{Du}^{1,2}$ \\ 1 College of Engineering, Huaqiao University, Quanzhou 362021, China; yongzhaodu@hqu.edu.cn; \\ Tel.: +86-188-1599-2396 \\ 2 Fujian Provincial Academic Engineering Research Centre in Industrial Intelligent Techniques and Systems, \\ Huaqiao University, Quanzhou 362021, China
}

Academic Editor: Vittorio M. N. Passaro

Received: 17 October 2016; Accepted: 23 November 2016; Published: 29 November 2016

\begin{abstract}
For asymmetric laser beams, the values of beam quality factor $\mathrm{M}_{x}^{2}$ and $\mathrm{M}_{y}^{2}$ are inconsistent if one selects a different coordinate system or measures beam quality with different experimental conditionals, even when analyzing the same beam. To overcome this non-uniqueness, a new beam quality characterization method named as $\mathrm{M}^{2}$-curve is developed. The $\mathrm{M}^{2}$-curve not only contains the beam quality factor $\mathrm{M}_{x}^{2}$ and $\mathrm{M}_{y}^{2}$ in the $x$-direction and $y$-direction, respectively; but also introduces a curve of $\mathrm{M}_{x \alpha}^{2}$ versus rotation angle $\alpha$ of coordinate axis. Moreover, we also present a real-time measurement method to demonstrate beam propagation factor $\mathrm{M}^{2}$-curve with a modified self-referencing Mach-Zehnder interferometer based-wavefront sensor (henceforth SRI-WFS). The feasibility of the proposed method is demonstrated with the theoretical analysis and experiment in multimode beams. The experimental results showed that the proposed measurement method is simple, fast, and a single-shot measurement procedure without movable parts.
\end{abstract}

Keywords: laser beam characterization; beam quality $\mathrm{M}^{2}$-curve; self-referencing interferometer wavefront sensor; fringe pattern analysis

\section{Introduction}

Laser beam characterization plays an important role for laser theoretical analysis, designs, and manufacturing, as well as medical treatment, laser welding, and laser cutting and other applications. Therefore, an effective evaluation parameter and its corresponding reliable measurement method for quantifying beam quality are necessary. Among various charactering laser beam quality approaches, the most commonly and widely used approach is the beam propagation factor, simply called 'beam quality $\mathrm{M}^{2}$ factor' which was first developed by Siegman [1,2] in 1990 . The $\mathrm{M}^{2}$ factor is defined as the ratio of the beam parameter product of an actual beam to that of an ideal Gaussian beam $\left(\mathrm{TEM}_{00}\right)$ at the same wavelength. That proposal was immediately adopted by the International Organization for Standardization (ISO). The standard draft, ISO/TC172/SC9/WG1, for laser beam characterization and its measurement method, was published in 1991 [3]. Now ISO published the latest version of $\mathrm{M}^{2}$ factor measurement Standard ISO11146-1/2/3 [4-6], which standardized laser beam characterization by defining all relevant quantities of laser beams including in measurement method instructions. Now $\mathrm{M}^{2}$ factor undoubtedly becomes an acceptable characterization parameter for beam quality characterization in the laser community [7-14].

The definition of the beam propagation ratio $\mathrm{M}^{2}$ for simple and general astigmatic beams and the instruction for its measurement can be found in the Standard ISO11146 [4-6]. The beam quality $\mathrm{M}^{2}$ factor is determined by the beam width as a function of propagation distance $(z)$ or propagation location of the test beams using hyperbolic fitting approaches. Here, the beam intensity distributions of the test beam measured with a moving CCD camera in various planes is suggested, which allows the 
determination of the beam widths by using second-order moments of the beam intensity distributions and hence the $\mathrm{M}^{2}$ value. However, using this scanning CCD-based method for $\mathrm{M}^{2}$ value is quite time-consuming due to the requirements of multiple measurements, consequently, it is unsuitable to characterize the fast dynamics of a laser beam system. In the past decades, some techniques [15-24] have been developed to measure the dynamic beam quality $\mathrm{M}^{2}$ factor, such as the Hartmann wavefront sensor [15,16], but it was shown to yield inaccurate results for multimode beams $[17,18]$. Other methods to perform $\mathrm{M}^{2}$ comply with ISO11146 techniques, for example, multi-plane imaging using distortion diffraction gratings [19], optical field reconstruction using modal decomposition [20,21], and spatial light modulator method [22]. The self-reference interferometer wavefront sensor (SRI-WFS) method $[23,24]$ is another effective method for real-time measurement of $\mathrm{M}^{2}$ factor which was developed recently. In general, SRI-WFS is based on a point diffraction interferometer, and its reference wave is generated by the pinhole diffraction with a pinhole filter and it no longer needs an artifacts reference beam, therefore, it is called "self-referencing" $[25,26]$. The SRI-WFS are usually used as a wavefront diagnosis tool for wavefront phase measurement. However, it cannot measure amplitude (or intensity) at the same time [25,26]. In Reference [24], a modified Mach-Zehnder point diffraction interferometer is developed for reconstructing complex amplitudes including in phase and amplitude (or intensity), and beam quality, $\mathrm{M}^{2}$ factor, can be calculated starting from the complex amplitude field of the test beams by using numerical method conforming to the ISO11146 standard [24].

For the asymmetric laser beams such as simple astigmatic beam and complex astigmatic beam [27,28], $\mathrm{M}_{x}^{2}$ and $\mathrm{M}_{y}^{2}$ are usually used to evaluate the beam quality in the $x$-direction and the $y$-direction, respectively [4-6]. However, we found that the values of $\mathrm{M}_{x}^{2}$ and $\mathrm{M}_{y}^{2}$ may change or are inconsistent if the coordinate axis is rotated. In other words, they are coordinate system dependent and change under different experimental conditions even when analyzing the same beam. Therefore, just using the simple parameters of $\mathrm{M}_{x}^{2}$ and $\mathrm{M}_{y}^{2}$ to characterize the beam quality of an asymmetric beam, such as a simple astigmatic beam and complex astigmatic beam, are not comprehensive or objective $[9,29]$. In references [30-32], a new beam characterization method, beam quality $\mathrm{M}^{2}$-matrix, is developed. It not only contains the beam quality terms, $\mathrm{M}_{x}^{2}$ and $\mathrm{M}_{y}^{2}$, to evaluate the beam quality in $x$-direction and $y$-direction, but also introduces another cross term, $\mathrm{M}_{x y}$, which is used to characterize the cross-relationship between $\mathrm{x}$-direction and y-direction [30,31]. The $\mathrm{M}^{2}$-matrix has more general physical meaning and wider application scope both for asymmetric beams and astigmatic beam [32].

In this paper, a new beam quality characterization method, beam quality $\mathrm{M}^{2}$-curve, for an asymmetric laser beam is developed. The $\mathrm{M}^{2}$-curve not only contains the beam quality factor $\mathrm{M}_{x}^{2}$ and $\mathrm{M}_{y}^{2}$ in the $x$-direction and $y$-direction, respectively; but also introduces a curve of $\mathrm{M}_{x \alpha}^{2}$ versus rotation angle $\alpha$ of coordinate axis. Moreover, we also present a real-time measurement method to demonstrate beam propagation factor $\mathrm{M}^{2}$-curve with a modified self-referencing Mach-Zehnder interferometer based-wavefront sensor (henceforth SRI-WFS). By using the SRI-WFS, a full characterization of laser beams both amplitude or intensity profile and wavefront phase are reconstructed from a single fringe pattern with Fourier transform (FT) based spatial phase modulation technology [33]. The beam quality $\mathrm{M}^{2}$-curve can be obtained starting from the complex field of the test laser beam by using the virtual caustic method conforming to the ISO standard method [4]. The feasibility of the proposed method is demonstrated with the theoretical analysis and experiment in asymmetric (Hermite-Gaussian-like mode) beams. The article is arranged as follows: The first section is the introduction. The theoretical analysis and derivation of SRI-WFS based complex amplitude reconstruction method is presented in Section 2. According to the Standard ISO11146, the formula derivation process for determining beam quality $\mathrm{M}^{2}$-curve starting from the reconstructed complex amplitude is showed in Section 3 . In Section 4 , the experimental results of beam quality $\mathrm{M}^{2}$-curve with the proposed SRI-WFS method are demonstrated. Finally, the conclusions are derived from this work. 


\section{Self-Referencing Interferometer Wavefront Sensor}

In this section, a new complex amplitude reconstruction method based on self-referencing Mach-Zehnder interferometer wavefront sensor (SRI-WFS) is presented. Firstly, the SRI-WFS experiment configuration is introduced. Secondly, we give the basic theory of complex amplitude method based on SRI-WFS, including in the theoretical derivation process and fringe pattern analysis method for reconstructing the complex amplitude of the test beam.

\subsection{Experiment Setup of SRI-WFS}

The experimental setup of SRI-WFS, which can be seen as a modified Mach-Zehnder radial shearing interferometer [34-36], is shown in Figure 1. This optical system consists of two beam-splitters, BS1 and BS2; a spatial filter, pinhole; two mirrors, M1 and M2; a two-lens positive telescope imaging systems which consisted of two lens, L1 and L2, with focal lengths of $f_{1}=100 \mathrm{~mm}$ and $f_{2}=300 \mathrm{~mm}$, respectively; and another two-lens positive telescope imaging systems which consisted of two lens, L3 and L4, with focal lengths of $f_{3}=300 \mathrm{~mm}$ and $f_{4}=100 \mathrm{~mm}$, respectively. A pinhole plate, functioning as a low-pass spatial filter, is placed at the common focal plane of Lens L1 and L2. The signal and reference waves formed interference pattern in the CCD plane. Here we define, $g=f_{2} / f_{1}=f_{3} / f_{4}$, and the magnification of SRI-WFS is defined as $G=g^{2}$. Compared to the tradition radial shearing interferometer configuration, in our experimental setup as shown in Figure 1, we made an improvement by simultaneously using a pinhole filter and a telescope system in each arm of SRI-WFS to generate a promising reference wave.

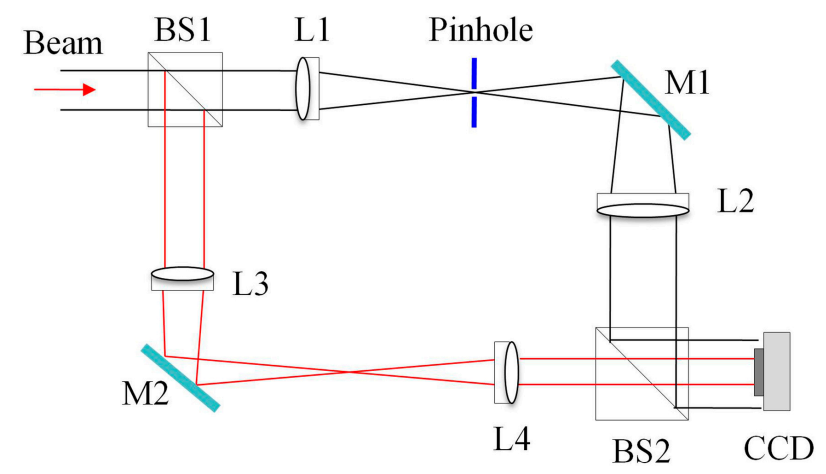

Figure 1. Experimental setup of SRI-WFS: BS1, BS2, beam-splitters, L1-L4, lens with the focal length $f_{1}=f_{4}=100 \mathrm{~mm}$ and $f_{2}=f_{3}=300 \mathrm{~mm}$, respectively; pinhole, spatial filtering plate with diameter $d_{\text {pin }}=25 \mu \mathrm{m} ; \mathrm{M} 1, \mathrm{M} 2$, reflection mirrors.

\subsection{Reconstruction of Complex Amplitude Field}

For simplicity, we denote the field of the incident test beam as,

$$
E(r, \varphi)=u(r, \varphi) \exp [i \phi(r, \varphi)]
$$

where $r$ and $\varphi$ are radial and circular coordinates, respectively; $u(r, \varphi)$ denotes the amplitude and $\phi(r, \varphi)$ is the phase information of incident test beam. As shown in Figure 1, the incident beam was split into two beams by the beam-splitter BS1, the reflected beam, and transmitted beam, which acted as signal wave and reference wave, respectively. The reflected beam travels along the invert telescope which consists of lens L3 and L4, and forms a constructed beam and served as a signal wave,

$$
E_{S}(g r, \varphi)=g \sqrt{R} \cdot u(g r, \varphi) \exp [i \phi(g r, \varphi)]
$$

Here, $g$ is the amplification factor of the invert telescope and it is defined as $g=f_{3} / f_{4} ; R$ in the reflectivity of BS1. The signal wave contains all the information (including in the amplitude $u(g r, \varphi)$ 
and the phase $\phi(g r, \varphi))$ of the tested beam $E(r, \varphi)$. The transmitted beam served as a reference wave which can be deduced by Fourier optics theory [37-40],

$$
E_{r}\left(\frac{r}{g}, \varphi\right)=\frac{\sqrt{1-R}}{g} \cdot u\left(\frac{r}{g}, \varphi\right) \exp \left[\mathrm{i} \phi\left(\frac{r}{g}, \varphi\right)\right] \otimes T\left(\frac{r}{g}, \varphi\right),
$$

where $u(r / g, \varphi)$ represents the amplitude of the reference wave; and $(r / g, \varphi)$ is the wavefront phase of the reference wave; $\otimes$ is the two-dimensional convolution operator. $T(r / g, \varphi)$ is the Fourier transform of the pinhole with diameter $d_{\text {pin }}$, and it is given by [40],

$$
T\left(\frac{r}{g}, \varphi\right)=\frac{\pi d_{p i n}^{2}}{4 \lambda f_{1}} \cdot 2 J_{1}\left[\frac{\pi d_{p i n} r}{s \cdot \lambda f_{1}}\right] /\left[\frac{\pi d_{p i n} r}{s \cdot \lambda f_{1}}\right] .
$$

Here $J_{1}$ is a first-order Bessel function of the first kind.

The signal wave $E_{s}(g r, \varphi)$ and the reference wave $E_{r}(r / g, \varphi)$ interfere in their superposition area $(g r, \varphi)$. Therefore, the fringe pattern produced by the signal wave $E_{s}(g r, \varphi)$ and the reference wave $E_{r}(r / g, \varphi)$ captured by the CCD can be expressed as,

$$
I(g r, \varphi)=\left|E_{r}\left(\frac{r}{g}, \varphi\right) \exp [\mathrm{i} \kappa(g r, \varphi)]+E_{s}(g r, \varphi)\right|^{2},
$$

where $\kappa$ denotes the linear carrier-frequency coming from an angle between the reference and signal waves, which is formed by slightly tilting beam-splitter BS2.

Considering the contracted signal wave and enlarged reference wave in the SRI-WFS system, we redefine the overlapping area, $(r g, \varphi)$, responding to the field of the signal wave, as a new coordinate domain $\left(r^{\prime}, \varphi\right)$. The field of signal wave can be shown as $\left(r^{\prime}, \varphi\right)$, and the reference wave is shown as $E\left(r^{\prime} / g^{2}, \varphi\right)$. Therefore, the Equation (5) can be rewritten as,

$$
I\left(r^{\prime}, \varphi\right)=\left|E_{r}\left(\frac{r^{\prime}}{G^{\prime}} \varphi\right) \exp \left[\mathrm{i} \kappa\left(r^{\prime}, \varphi\right)\right]+E_{s}\left(r^{\prime}, \varphi\right)\right|^{2},
$$

where $G=g^{2}$ is the magnification of the SRI-WFS. For simplicity, we define the overlapping area $(x / G, y / G)$ as new coordinate domain $(x, y)$ and its intensity distribution in the CCD plane can be written into a general form of the fringe pattern, as follows,

$$
i(x, y) \propto a(x, y)+b(x, y) \cos \left[2 \pi\left(\kappa_{x} x+\kappa_{y} y\right)+\phi(x, y)\right],
$$

where $a(x, y) \propto E_{s}{ }^{2}(x, y)+E_{r}{ }^{2}(x / G, y / G)$ is the background intensity, and $b(x, y) \propto 2 u_{s}(x, y) u_{r}(x / G, y / G)$ is the modulation intensity of fringe pattern; $\kappa_{x}$ and $\kappa_{y}$ are the carrier-frequency components along $x$-direction and $y$-direction, respectively. Therefore, the complex amplitude of modulation (CAM) function [41] of the interferogram as shown in Equation (7) can be easily extracted by spatial phase modulation (SPM) technology, proposed firstly by M. Takeda [33]. The CAM function contains both information of the wavefront phase $\phi(x, y)$ and amplitude $E_{s}(x, y)$ of the test beams.

Taking the Fourier transform in two-dimension for the interference pattern $[33,42,43]$,

$$
\begin{aligned}
I(X, Y) & =A(X, Y)+[\boldsymbol{F T}\{\exp [-\mathrm{i} \phi(x, y)]\} \otimes B(X, Y)] \otimes \delta\left(X-\kappa_{x}, Y-\kappa_{y}\right) \\
& +[\boldsymbol{F T}\{\exp [\mathrm{i} \phi(x, y)]\} \otimes B(X, Y)] \otimes \delta\left(X+\kappa_{x}, Y+\kappa_{y}\right),
\end{aligned}
$$

where $\boldsymbol{F T}\{\}$ denotes the Fourier transform, $X$ and $Y$ are the spatial frequency variables, and $\delta(X, Y)$ is the Dirac's delta function, $\mathrm{A}(X, Y)$ and $\mathrm{B}(X, Y)$ presents the frequency-spectrum of $\mathrm{a}(x, y)$ and $\mathrm{b}(x, y)$, respectively. The second and third term in Equation (8) are two Dirac's functions placed in $\left(\kappa_{x}, \kappa_{y}\right)$ and $\left(\kappa_{x}, \kappa_{y}\right)$ around the low spatial frequency $A(X, Y)$, respectively. By using of a band pass filter, it is possible to extracts the Dirac delta function $\delta\left(X-\kappa_{x}, Y-\kappa_{y}\right)$. Then, tacking the inverse 
two-dimension Fourier transform of the Dirac's delta function $\delta\left(X-\kappa_{x}, Y-\kappa_{y}\right)$, that is, obtaining the CAM function, as follows [24],

$$
c(x, y)=\frac{1}{2} b(x, y) \propto u_{s}(x, y) u_{r}\left(\frac{x}{G}, \frac{y}{G}\right) \exp [-\mathrm{i} \phi(x, y)] .
$$

In this equation, it can be seen that it is similar to the description of complex amplitude of the test wave which is expressed in Equation (1). If the magnification $G$ of SRI-WFS is large enough, the term $E_{r}(x / G, y / G)$ will approach to a uniform plane wave, that is, the amplitude tends to $u_{r}(0,0)$ and the phase component tends to an ideal plane $\phi(0,0)$. Taking into account the amplitude is a relative value in practical application, and the coefficient $u_{r}(0,0)$ in the Equation (9) can be rewritten as a constant $\mathrm{C}$. Thus, the complex amplitude distribution of test laser beam can be expressed as [24],

$$
E(x, y) \propto \lim _{G \rightarrow \infty} c(x, y)=C \cdot u_{s}(x, y) \exp [-\mathrm{i} \phi(x, y)]
$$

\section{Determination of $\mathbf{M}^{2}$-Curve}

\subsection{Measurement Method for Beam Quality Factor $M^{2}$ Based on the Complex Amplitude Distribution [24]}

Once the complex amplitude field $E(x, y, 0)$ of the test laser beam is obtained, according to the diffraction integral theory $[37,44]$, the complete complex amplitude fields $E(x, y, z)$ at $z$ planes after propagation distance $z$ can be calculated using a double fast Fourier transform algorithm [44],

$$
E(x, y, z)=\boldsymbol{F} \boldsymbol{T}^{-1}\left\{\boldsymbol{F} \boldsymbol{T}\{E(x, y, 0)\} \exp \left\{\operatorname{ikz}\left[1-\frac{\lambda^{2}}{2}\left(\eta_{x}^{2}+\eta_{y}^{2}\right)\right]\right\}\right\}
$$

where $z$ is the propagation distance, $\mathrm{k}$ is wave number, $\lambda$ is wavelength of test laser beam, $\eta_{x}=x / \lambda_{z}$, $\eta_{y}=y / \lambda_{z}$ are the spatial frequencies, respectively. The direct $(\boldsymbol{F T})$ and inverse $\left(\boldsymbol{F} \boldsymbol{T}^{-1}\right)$ Fourier transform are calculated using a Fast Fourier Transform algorithm. Furthermore, in accordance with the ISO standard [4-6], the complex amplitude fields $E(x, y, z)$ can be used to determine the beam parameters relevant for beam propagation, i.e., beam widths $w_{x}(z)$ and $w_{y}(z)$. Performing a hyperbolic fit to the beam width along the beam transmission axis,

$$
w_{x, y}^{2}(z)=a_{x, y} z^{2}+b_{x, y} z+c_{x, y}
$$

where $w_{x, y}$ is the beam width in the $x$-direction or $y$-direction; $a_{x, y}, b_{x, y}$ and $c_{x, y}$ are the hyperbolic fitting coefficients, and the subscript $x$ and $y$ correspond to the values along the $x$-direction or $y$-direction, respectively. Then the waist radius $w_{x 0, y 0}$, divergence half angle $\theta_{x, y}$ and beam propagation factor $\mathbf{M}_{x, y}^{2}$ can be calculated from the following expressions [4],

$$
\begin{gathered}
w_{x 0, y 0}^{2}=c_{x, y}-\frac{b_{x, y}^{2}}{4 a_{x, y}} ; \\
\theta_{x 0, y 0}^{2}=a ; \\
\mathrm{M}_{x, y}^{2}=\frac{\pi}{\lambda} \cdot \sqrt{a_{x, y} c_{x, y}-\frac{b_{x, y}^{2}}{4}} .
\end{gathered}
$$

According to above analysis (Sections 2.1, 2.2 and 3.1), as long as the phase $\phi(x, y)$ and the amplitude $u(x, y)$ of the test laser beam are obtained, it is possible to calculate the beam quality $\mathrm{M}^{2}$ factor, specifically, the values of $\mathrm{M}_{x}^{2}$ and $\mathrm{M}_{y}^{2}$ along $x$-direction and $y$-direction of the test beams, respectively. 


\section{2. $M^{2}$-Curve}

The output beam of a multimode-laser stable cavity with rectangular geometry in many transverse modes can be written as an incoherent superposition of Hermite-Gaussian (HG) beams [45,46]. Usually the spatial modes are separable; the complex amplitude field distribution of HG modes, at the plane $z=0$, can be written as [45],

$$
\mathrm{HG}_{m n}(x, y, 0)=\frac{1}{\sqrt{w_{x 0} w_{y 0}}} \sqrt{\frac{2}{2^{m+n} \pi m ! n !}} \mathrm{H}_{m}\left[\frac{\sqrt{2}}{w_{x 0}} x\right] \exp \left[-\frac{x^{2}}{w_{x 0}^{2}}\right] \times \mathrm{H}_{n}\left[\frac{\sqrt{2}}{w_{y 0}} y\right] \exp \left[-\frac{y^{2}}{w_{y 0}^{2}}\right],
$$

where the phase term is ignored, $w_{0}$ is the waist radius of the fundamental mode $\mathrm{HG}_{00}$; and $\mathrm{H}_{m}$ and $\mathrm{H}_{n}$ denotes the Hermite-polynomial of order $m$ in $x$-direction and order $n$ in $y$-direction, respectively. HG modes are one set of orthogonal eigenfunctions of the scalar Helmholtz wave equation. Due to the completeness of this eigenfunction set, an arbitrary transverse wave field $E(x, y, z)$ can be expanded into a superposition of HG modes [46],

$$
E(x, y, z)=\sum_{m=0}^{\infty} \sum_{n=0}^{\infty} c_{m n} \mathrm{HG}_{m n}(x, y, z)
$$

with

$$
c_{m n}=\iint_{\infty} \mathrm{HG}_{m n}^{*} E(x, y, z) \mathrm{d} x \mathrm{~d} y,
$$

where the asterisk denotes complex conjugation operator; $c_{m n}$ is the complex-valued expansion coefficients, $c_{m n}=C_{m n} \exp \left(\varphi_{m n}\right)$, including in the modal amplitude $C_{m n}=\left|c_{m n}\right|$ and phase $\phi_{m n}=\operatorname{angle}\left(c_{m n}\right)$.

As the analysis above, generally, the intensity profile of the output beam emitted by a multimode stable cavity is non-rotational symmetric or asymmetric beams, as shown in Figure 2. According to Sigman's theories [1,2], the quality of the laser beam is evaluated by $\mathrm{M}_{x}^{2}$ in $x$-direction and $\mathrm{M}_{y}^{2}$ in $y$-direction. However, we found that the values of $\mathrm{M}_{x}^{2}$ and $\mathrm{M}_{y}^{2}$ are non-unique if we rotate coordinate the axis in an astigmatic laser beam. Consequently, just using the simple parameters $\mathrm{M}_{x}^{2}$ and $\mathrm{M}_{y}^{2}$ to characterize the beam quality of an asymmetric beam is not comprehensive or objective $[9,29,30]$.

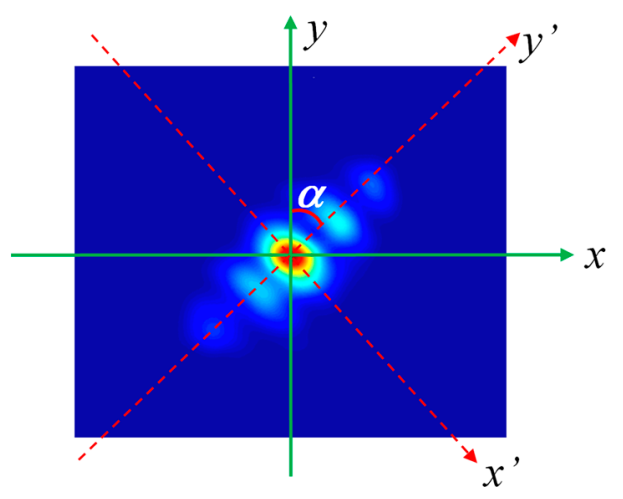

Figure 2. Asymmetric beam intensity distribution in the laboratory coordinate system.

Similarly to the traditional definitions form of the beam widths in the general astigmatic beam [4], we have

$$
w_{x z}^{2}=\frac{4 \iint_{-\infty}^{+\infty} I(x, y, z)(x-\bar{x})^{2} \mathrm{~d} x \mathrm{~d} y}{\iint_{-\infty}^{+\infty} I(x, y, z) \mathrm{d} x \mathrm{~d} y}
$$

and

$$
w_{y z}^{2}=\frac{4 \iint_{-\infty}^{+\infty} I(x, y, z)(y-\bar{y})^{2} \mathrm{~d} x \mathrm{~d} y}{\iint_{-\infty}^{+\infty} I(x, y, z) \mathrm{d} x \mathrm{~d} y}
$$


and

$$
w_{x y z}=\frac{4 \iint_{-\infty}^{+\infty} I(x, y, z)(x-\bar{x})(y-\bar{y}) \mathrm{d} x \mathrm{~d} y}{\iint_{-\infty}^{+\infty} I(x, y, z) \mathrm{d} x \mathrm{~d} y}
$$

and

$$
w_{r z}^{2}=\frac{4 \iint_{-\infty}^{+\infty} I(x, y, z)\left[(x-\bar{x})^{2}+(y-\bar{y})^{2}\right] \mathrm{d} x \mathrm{~d} y}{\iint_{-\infty}^{+\infty} I(x, y, z) \mathrm{d} x \mathrm{~d} y}
$$

where $\bar{x}$ and $\bar{y}$ are the centroid coordinates of the power density distribution which defined as the first-order moment of the power density distribution of the test beams. Therefore, the corresponding beam divergence half angles are given by [4],

$$
\begin{gathered}
\theta_{x}=\lim _{z \rightarrow \infty} \frac{w_{x z}}{z} \\
\theta_{y}=\lim _{z \rightarrow \infty} \frac{w_{y z}}{z} \\
\theta_{x y}=\lim _{z \rightarrow \infty} \frac{w_{x y z}}{z}=\lim _{z \rightarrow \infty} \frac{w_{y x z}}{z} \\
\theta_{r}=\lim _{z \rightarrow \infty} \frac{w_{r z}}{z}
\end{gathered}
$$

As shown in Figure 2, assuming the rotation angle of the coordinate axis is $\alpha$, we have

$$
x_{\alpha}=x \cos \alpha-y \sin \alpha, y_{\alpha}=x \sin \alpha+y \cos \alpha
$$

Therefore, the beam widths and the beam divergence half angles of the original and rotated beam (with a rotation angle $\alpha$ ) have the following relationships which are given by the Equations (28)-(31), respectively; as following [30,32],

$$
\begin{gathered}
w_{x z \alpha}^{2}=w_{x z}^{2} \cos ^{2} \alpha+w_{y z}^{2} \sin ^{2} \alpha \\
w_{y z \alpha}^{2}=w_{x z}^{2} \sin ^{2} \alpha+w_{y z}^{2} \cos ^{2} \alpha \\
\theta_{x z \alpha}^{2}=\theta_{x z}^{2} \cos ^{2} \alpha+\theta_{y z}^{2} \sin ^{2} \alpha \\
\theta_{y z \alpha}^{2}=\theta_{x z}^{2} \sin ^{2} \alpha+\theta_{y z}^{2} \cos ^{2} \alpha
\end{gathered}
$$

Utilizing the Equations (28)-(31), it is easy to derive the following expressions,

$$
\begin{gathered}
w_{x \alpha}^{2}(z)+w_{y \alpha}^{2}(z)=w_{r}^{2}(z) \\
\theta_{x \alpha}^{2}+\theta_{y \alpha}^{2}=\theta_{r}^{2} \\
\frac{\pi^{2}}{\lambda^{2}}\left(w_{x \alpha}^{2}+w_{y \alpha}^{2}\right)\left(\theta_{x \alpha}^{2}+\theta_{y \alpha}^{2}\right)=\frac{\pi^{2}}{\lambda^{2}} w_{r}^{2} \theta_{r}^{2} .
\end{gathered}
$$

In this case, we can derive a new beam propagation ratio $\mathrm{M}^{2}(\alpha)$ with rotation angle $\alpha$ to characterize the rotated beam and it can be given as,

$$
\mathrm{M}^{2}(\alpha)=\frac{\pi}{\lambda} w_{x 0 \alpha} \theta_{x \alpha},
$$

where $w_{x 0 \alpha}$ and $\theta_{x \alpha}$ are waist radius and divergence half angle in the $x$-direction with a rotation angle $\alpha$ of coordinate axis, respectively. $\mathrm{M}^{2}$ can obtain different values if the rotation angle $\alpha$ charges with the coordinate axis, that is, a track of the $\mathrm{M}^{2}$ values versus the rotation angle $(\alpha)$ of the beam coordinate axis will be form and named $\mathrm{M}^{2}$-curve which is unique to a specific tested beam. It can 
be seen from the Equation (35) that the $\mathrm{M}^{2}$-curve takes into account changes in angle and can give a track of the $\mathrm{M}^{2}$ values which is compared to the rotation angle $\alpha$ of the beam coordinate axis. That is, the $\mathrm{M}^{2}$-curve contains all the values of beam quality $\mathrm{M}^{2}$ in all directions. The traditional $\mathrm{M}^{2}$ factor $\mathrm{M}_{x}^{2}$ in $x$-direction and $\mathrm{M}_{y}^{2}$ in $y$-direction are just special cases of the $\mathrm{M}^{2}$-curve in a special direction of the tested beam, precisely in two orthogonal directions ( $x$-axis and $y$-axis). Therefore, the $\mathrm{M}^{2}$-curve has a more comprehensive physical meaning and is more objective for characterizing the beam quality because it can be uniquely determined for a particular tested beam. In other words, there is a one-to-one mapping relationship between a particular tested beam and its sharp of $\mathrm{M}^{2}$-curve. According to the above analysis in Sections 2 and 3, we can summarize the flow chart of the $\mathrm{M}^{2}$-curve determined by the SRI-WFS method, as shown in Figure 3.

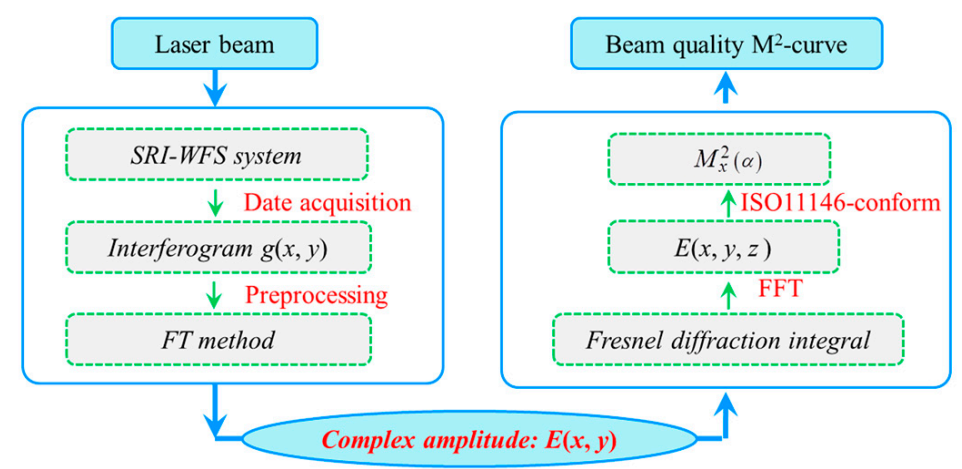

Figure 3. The flow chart of beam quality $\mathrm{M}^{2}$-curve determined by the proposed SRI-WFS method.

\section{Experiment Results and Discussions}

The beams from a diode pump solid state laser (DPSSL) with $532 \mathrm{~nm}$ wavelength are used as test beam. The excitation of mode mixture in the DPSSL are Hermite-Gaussian-like modes (shorted as TELM $_{\mathrm{mn}}$ ) is achieved by adjusting the tilt angle of the cavity mirrors [45]. The experiment setup of SRI-WFS for completely reconstructing the complex amplitude field of a laser beam is depicted in Figure 1, and it has been demonstrated that it can be used for beam quality measurements in real-time and the details of SRI-WFS system can be seen in [24]. Two invert telescopes with telescope factor $g=3$, consisted of achromatic lens L1-L4 with focal length $f_{1}=f_{4}=100 \mathrm{~mm}$ and $f_{2}=f_{3}=300 \mathrm{~mm}$, respecting the magnification $G=9$ of SRI-WFS. A pinhole plate with diameter $d_{\text {pin }}=25 \mu \mathrm{m}$ is used to generate a reference wave. A CCD camera, model MVIC-II-1MM, with $1024 \times 1280$ pixels and 5.2 micron in each pixel, is positioned at the imaging plane to record interferogram in real time. The CCD sampled-data are sent to the personal computer system (PC) for further processing.

Adjusting a slight tilt angle in the cavity mirrors, we can obtain different mix-mode beam outputs whose intensity profiles are similar to the Hermite-Gaussian mode in $\mathrm{TEM}_{\mathrm{mn}}$. For the convenience of the following analysis, we denote these mix-mode beams as Hermite-Gaussian-like modes in TELM $\mathrm{mn}_{\mathrm{m}}$ to distinguish with the pure Hermite-Gaussian modes in $\mathrm{TEM}_{\mathrm{mn}}$. As shown in Figure 4, three intensity profiles corresponding to Hermite-Gaussian-like modes in TELM ${ }_{00}, \mathrm{TELM}_{20}$, and TELM 40 , respectively, are used as the test beams in our experiments. Corresponding interferograms formed with SRI-WFS are shown in Figure 5 (up row). The reconstructed intensity profiles (middle row) and the reconstructed wrapped phase distribution (low row) of the test beams are also shown in Figure 5. Each reconstructed distributions of amplitude and phase for the test beams are extracted from a single interferogram according to Equations (7)-(10). Considering the limited of conditions in our laboratory, we only investigate the intensity distribution for comparative analysis. For a quantitative comparison of the measurement and reconstruction intensity profile, two-dimension cross-correlation coefficient $C_{c}$ is used as a metric [24]. Here $C_{c}$ is defined as the cross-correlation between the images of measurement intensity distribution $I_{M}(x, y)$ and reconstruction intensity distribution $I_{R}(x, y)$, and $C_{c}=1$ is indicated that the measured intensity profile and reconstructed intensity profiles match perfectly. In our 
experiment, the $\mathrm{C}_{\mathrm{c}}$ of the reconstructed and measurement intensities for the Hermite-Gaussian-like modes in TELM 00, TELM $_{20}$ and TELM 40 are 0.999, 0.998 and 0.997, respectively.
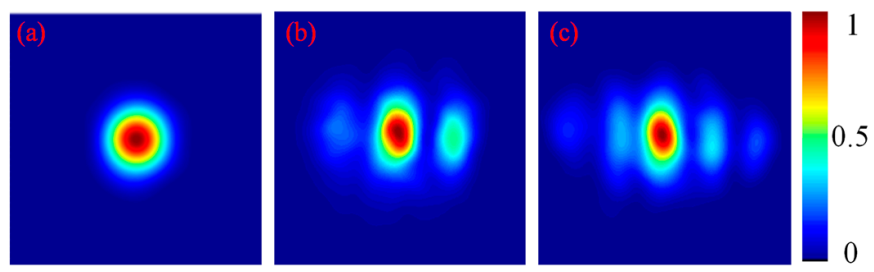

Figure 4. Measured intensity profiles of Hermite-Gaussian-like modes in (a) TELM 00 ; (b) TELM 20 and (c) TELM $_{40}$.
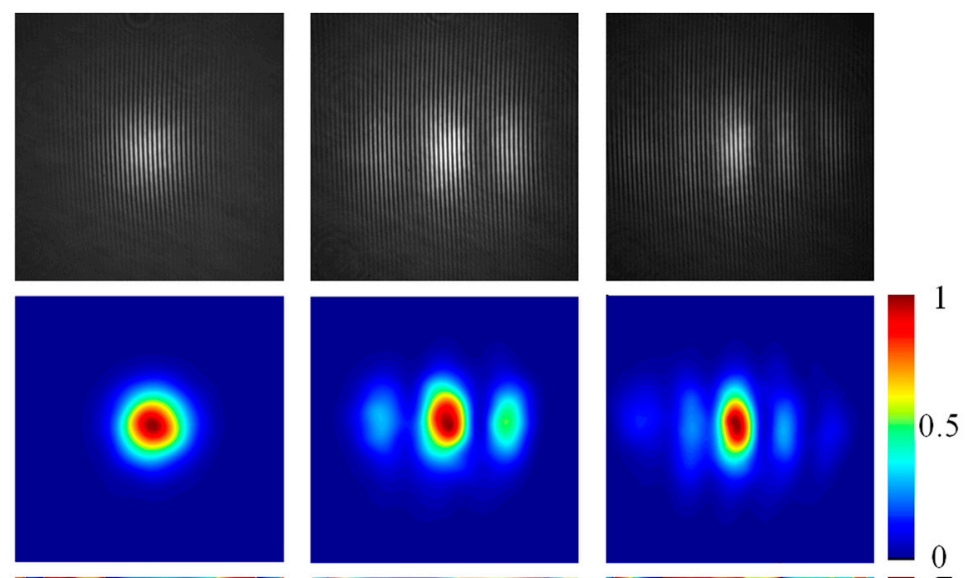

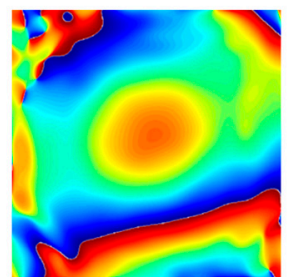

(a)

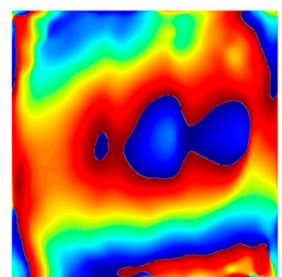

(b)

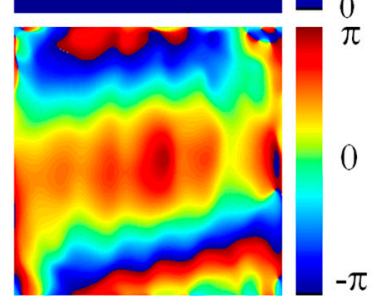

(c)

Figure 5. The captured interferogram, reconstructed intensity (a.u.), and wrapped phase maps of Hermite-Gaussian-like modes in (a) TELM 00 ; (b) TELM 20 and (c) TELM 40 . Interferogram (up row), reconstructed intensity profile (middle row), and the reconstructed phase (low row) of the test beams.

There are several causes for the deviation of the reconstruction intensity and the measured intensity: (1) optical components such as inaccurate errors of telescope system and misalignment in the SRI-WFS system lead to beam distortion; (2) the intensity of the reference wave hardly becomes completely flat with the limited magnification of SRI-WFS in practice; (3) filtering error in the Fourier transform method for interferogram analysis; etc. As long as reconstructing the complex amplitude field $E(x, y, 0)$ of the test beams, the field $E(x, y, z)$ at different plane of $z$-axis direction (with different propagation length $z$ ) can be determined easily according to the Equation (11), and the intensities distribution $I(x, y, z)=|E(x, y, z)|^{2}$ are also determined uniquely. According to the Standard ISO11146, the beam width $w_{x z}$ can be determined by the second moment of the obtained intensity distributions, as described in Equations (19)-(22). The beam width $w_{x z}$ of test beams in $x$-direction of Hermite-Gaussian-like modes TELM $00, \mathrm{TELM}_{20}$, and TELM 40 can be calculated directly from the intensity distribution $I(x, y, z)$ at different propagation location $z$. Moreover, according to the Equations (13) and (28)-(31), the beam widths $w_{x z \alpha}$ and the beam divergence half angles $\theta_{x z \alpha}$ with different rotation angle $\alpha$ of the coordinate axis also can be obtained. Figure 6 shows the results of the beam width $w_{x z \alpha}$ in the $x$-direction of Hermite-Gaussian-like modes (a) TELM 00 ; (b) TELM 20 
and (c) TELM $_{40}$ versus propagation distance $\mathrm{z}$ and the different rotation angle $\alpha$ of coordinate axis. Here, the rotation angles $\alpha$ are settled to $10,20,30,40,50,60,70,80$ and $90^{\circ}$; respectively.

It can be seen from Figure 6 that with the different rotation angle $\alpha$, for example, $\alpha=10,20,30,40$, $50,60,70,80$, and $90^{\circ}$; the beam widths $w_{x}$, the beam waist radius $w_{x 0}$, beam divergence half angles $\theta_{x}$, and waist positions $z_{0}$ of the tested beams are all very different. It is further evidenced by the results of the normalized waist radius $w_{x \alpha}\left(z_{0}\right)$ and beam divergence half angles $\theta_{x}$ in the $x$-directions for Hermite-Gaussian-like modes (TELM $00, \mathrm{TELM}_{20}$, and TELM 40 ) versus rotation angle of coordinate axis $\alpha$, as shown in Figure 7. That is, with the changing rotation angle of the coordinate axis of the tested beam in actual measurement, the waist radius $w_{x 0}$ and divergence half angle $\theta_{x}$ are ever-changes and these different will directly result in the non-unique of the values of $\mathrm{M}^{2}$ factor. Obviously, it is consistent with the previous analysis and the motivation of this paper. Moreover, it also indicates that using the simple $\mathrm{M}^{2}$ factor, $\mathrm{M}_{x}^{2}$ in $x$-direction and $\mathrm{M}_{y}^{2}$ in $y$-direction just two special cases (in the two orthogonal directions) of the test beams but missing the most of information in the other random rotation angles $\alpha$ of the test beams.
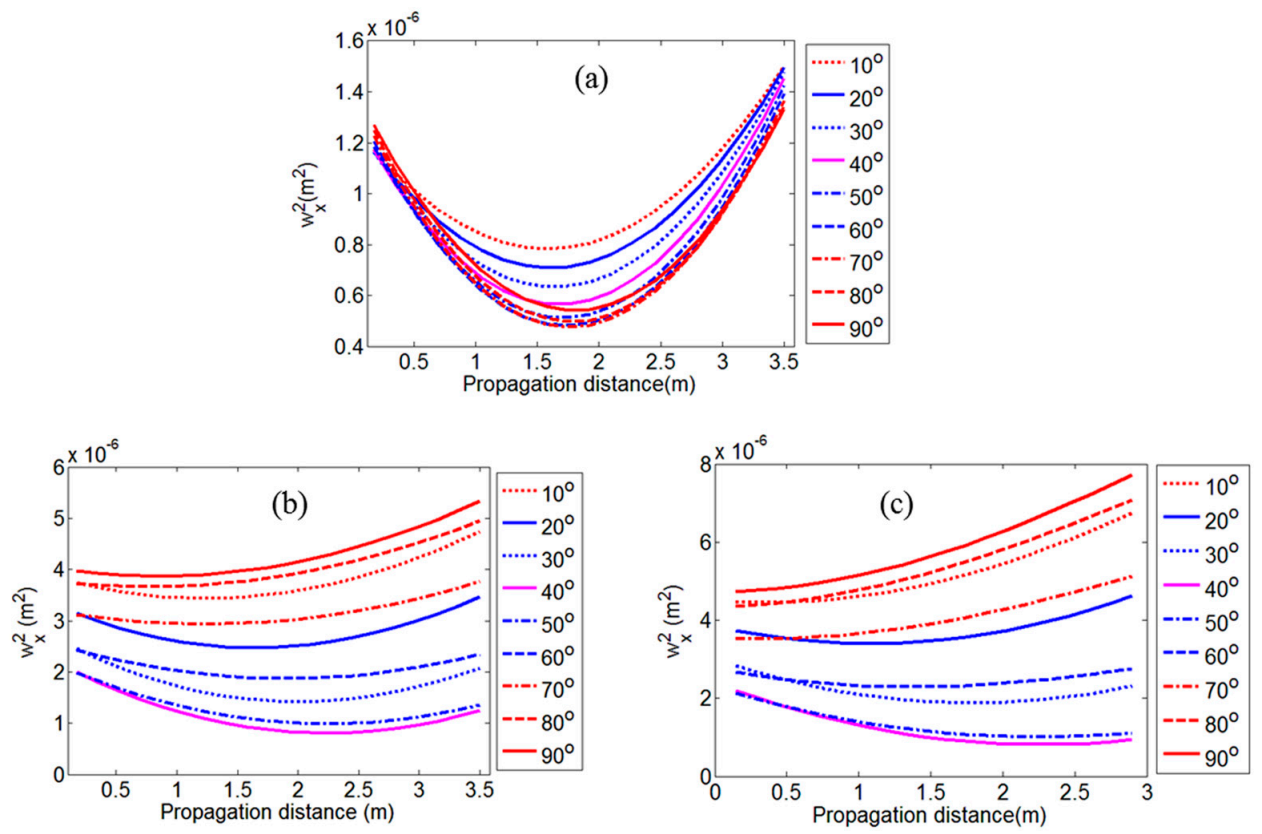

Figure 6. The beam widths $w_{x}$ in $x$-direction of Hermite-Gaussian-like modes (a) TELM 00 ; (b) TELM 20 ; and (c) TELM 40 versus propagation distance. The rotation angle $\alpha$ of the coordinate axis is 10, 20, 30, $40,50,60,70,80,90^{\circ}$, respectively.

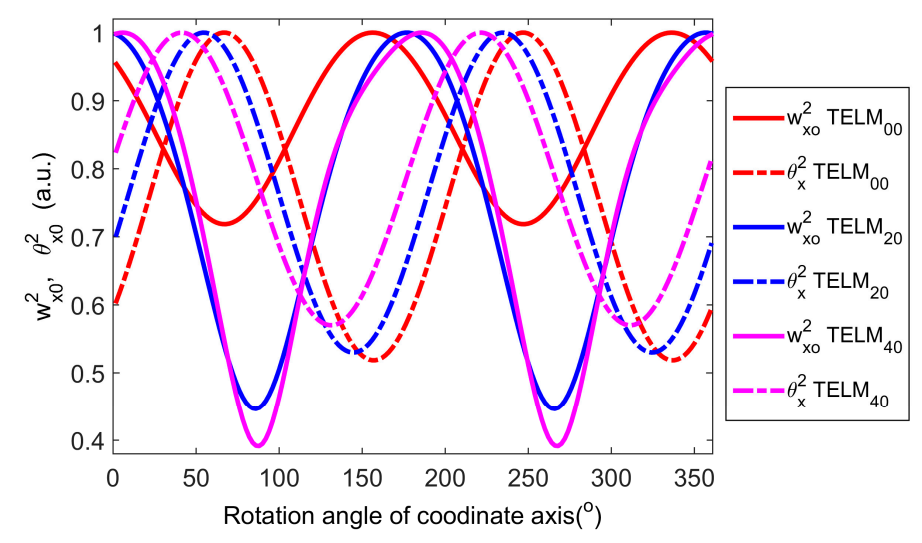

Figure 7. Normalized waist radius $\mathrm{w}_{x 0}$ and divergence half angle $\theta_{x}$ in $x$-directions for HermiteGaussian-like modes $\left(\mathrm{TELM}_{00}, \mathrm{TELM}_{20}\right.$, and TELM 40 ) versus rotation angle of coordinate axis. 
The results of the $\mathrm{M}^{2}$-curves of the test beams (Hermite-Gaussian-like modes TELM 00, TELM $_{20}$, and $\mathrm{TELM}_{40}$ ) are shown in Figure 8. Note that all the $\mathrm{M}^{2}$-curves for Hermite-Gaussian-like modes TELM $_{00}$, TELM $_{20}$, and TELM 40 are asymmetric. The $\mathrm{M}^{2}$-curve is maybe a circle, an ellipse, or an 8-shaped pattern. Moreover, the length values, from the original point to the cross-point at the curve, denote the $\mathrm{M}^{2}$ values along this rotation angle $\alpha$. For example, as shown in Figure 8, the values of the test beams, Hermite-Gaussian-like modes TELM 00, TELM $_{20}$ and TELM $_{40}$ along $\alpha=0$ direction, which corresponding to the traditional $\mathrm{M}^{2}$ factor $\mathrm{M}_{x}^{2}$ along $x$-direction of the tested beams, are 1.06, 2.62 and 3.72, respectively. Moreover, when the rotation angle $\alpha$ is equal to $90^{\circ}$, corresponding to $\mathrm{M}_{y}^{2}$ along the $y$-direction for the tradition $\mathrm{M}^{2}$ factor. In this case, the values of $\mathrm{M}_{y}^{2}$ are 1.08 for $\mathrm{TELM}_{00}, 1.27$ for $\mathrm{TELM}_{20}$, and 1.37 for TELM $_{40}$, respectively. For comparative analysis, we also measured the beam propagation factor $M^{2}$ values using the traditional ISO11146 standard based beam quality analyzer (BQA) developed in our previous work [25], and the results are shown in Table 1 . It shows that the beam quality $\mathrm{M}^{2}$ factors with ISO11146 standard based method are $\mathrm{M}_{0 x}^{2}=1.04, \mathrm{M}_{2 x}^{2}=2.54$ and $\mathrm{M}_{4 x}^{2}=3.56$ along the $x$-direction and $\mathrm{M}_{0 y}^{2}=1.06, \mathrm{M}_{2 y}^{2}=1.20$ and $\mathrm{M}_{4 y}^{2}=1.32$ along the $\mathrm{y}$-direction for Hermite-Gaussian-like modes $\mathrm{TELM}_{00}, \mathrm{TELM}_{20}$, and TELM 40 , respectively. Table 1 also shows the measurement errors between the $\mathrm{M}^{2}$ factor values of Hermite-Gaussian-like modes TELM $\mathrm{T}_{00}, \mathrm{TELM}_{20}$, and TELM 40 with the proposed SRI-WFS method and the ISO1116 standard based BQA. The maximum measured deviation of the two methods are $4.49 \%$ for $\mathrm{M}_{x}^{2}$ and $5.83 \%$ for $\mathrm{M}_{y}^{2}$ along $x$-axis and $y$-axis, respectively. It can be seen from Table 1 that the results of both measurement methods are well in agreement.

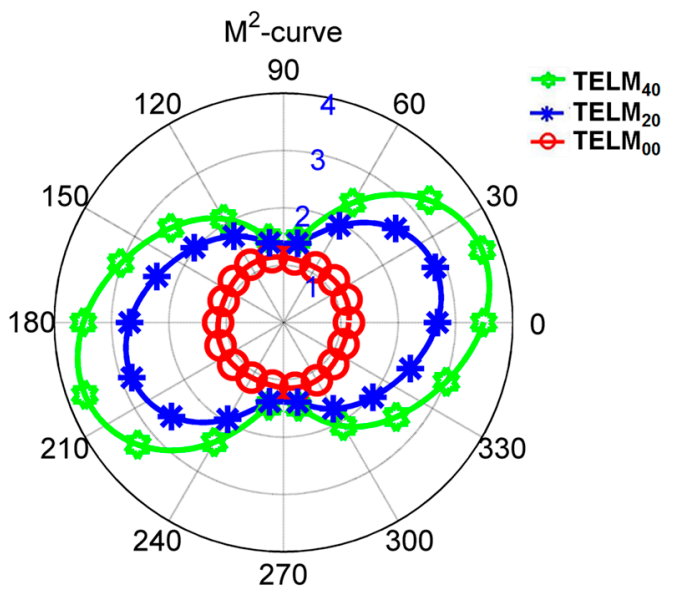

Figure 8. $\mathrm{M}^{2}$-curves of Hermite-Gaussian-like modes in $\mathrm{TELM}_{00}, \mathrm{TELM}_{20}$, and TELM 40 . Here, the numbers $1,2,3$, and 4 are the circular scale for the $\mathrm{M}^{2}$-curve.

Table 1. The measurement results of $\mathrm{M}^{2}$ factor for Hermite-Gaussian-like modes $\mathrm{TELM}_{00}, \mathrm{TELM}_{20}$, and TELM $_{40}$ with the proposed SRI-WFS method and the ISO1116 standard based beam quality analyzer.

\begin{tabular}{cccccccc}
\hline \multirow{2}{*}{ Beam Quality } & \multicolumn{3}{c}{$\mathbf{M}_{\boldsymbol{x}}^{\mathbf{2}}$} & & \multicolumn{3}{c}{$\mathbf{M}_{\boldsymbol{y}}^{\mathbf{2}}$} \\
\cline { 2 - 3 } & TELM $_{\mathbf{0 0}}$ & $\mathbf{T E L M}_{\mathbf{2 0}}$ & $\mathbf{T E L M}_{\mathbf{4 0}}$ & & $\mathbf{T E L M}_{\mathbf{0 0}}$ & $\mathbf{T E L M}_{\mathbf{2 0}}$ & $\mathbf{T E L M}_{\mathbf{4 0}}$ \\
\hline SRI-WFS & 1.06 & 2.62 & 3.72 & & 1.08 & 1.27 & 1.37 \\
ISO1116 method & 1.04 & 2.54 & 3.56 & & 1.06 & 1.20 & 1.32 \\
Errors (\%) & $1.96 \%$ & $3.15 \%$ & $4.49 \%$ & & $1.89 \%$ & $5.83 \%$ & $3.79 \%$ \\
\hline
\end{tabular}

As shown in Figure 8, the $\mathrm{M}^{2}$-curve include in all $\mathrm{M}^{2}$ information (including in the traditional $\mathrm{M}^{2}$ factor values) in all random rotation angle $\alpha$ of the coordinate axis. From this sense, $\mathrm{M}^{2}$-curve is an extension method for characterization of beam quality. Moreover, its shape will be uniquely determined for a particular test beam. Consequently, it provides a more comprehensive and more objective physical meaning for actual applications. 


\section{Conclusions}

The non-uniqueness of characterization of laser beam quality with the traditional $\mathrm{M}^{2}$ factor can be overcome by using the $\mathrm{M}^{2}$-curve. The $\mathrm{M}^{2}$-curve has more general physical meaning and wider application scope both for asymmetric beams and astigmatic beams. The $\mathrm{M}^{2}$-curve not only contains the beam quality terms, $\mathrm{M}_{x}^{2}$ and $\mathrm{M}_{y}^{2}$, to evaluate the beam propagation quality in the $x$-direction and $y$-direction, respectively; but also introduces a curve of $\mathrm{M}_{x \alpha}^{2}$ which is used to characterize beam propagation factor versus the rotation angle $\alpha$ of coordinate axis. Moreover, the measurement method of the $\mathrm{M}^{2}$-curve based on modified SRI-WFS is also put forward which is used to demonstrate the potential of the proposed method, $\mathrm{M}^{2}$-curve, for charactering asymmetric beams.

Acknowledgments: This work is supported by the grants from National Natural Science Foundation of China (No. 61605048), Natural Science Foundation of Fujian Province, China (No. 2016J01300), Fujian Scientific Research Platform for Innovation, China, (No. 2013H2002), and the Scientific Research Funds of Huaqiao University (No. 15BS413). Yongzhao Du thanks Guoying Feng (Sichuan University, China) for supporting this work and providing helpful discussion, and Max Wiedmann for improving the writing and expression in the manuscript.

Author Contributions: Yongzhao Du conceived and designed the experiments, performed the experiments, analyzed the data and wrote the paper.

Conflicts of Interest: The authors declare no conflict of interest.

\section{References}

1. Siegman, A.E. New developments in laser resonators, in Optical Resonators. Proc. SPIE 1990, 1224, 2-14.

2. Siegman, A.E. Defining, measuring, and optimizing laser beam quality. Proc. SPIE 1993, 1868, 2-13.

3. International Organization for Standardization. Terminology and Test Methods ISO/TC172/SC/WG1; ISO: Geneva, Switzerland, 1991.

4. International Standardization for Standardization. ISO 11146-1 Lasers and Laser-Related Equipment-Test Methods for Laser Beam Widths, Divergence Angles and Beam Propagation Ratios-Part 1: Stigmatic and Simple Astigmatic Beams; ISO: Geneva, Switzerland, 2005.

5. International Standardization for Standardization. ISO 11146-2, Lasers and Laser-Related Equipment-Test Methods for Laser Beam Widths, Divergence Angles and Beam Propagation Ratios-Part 2: General Astigmatic Beams; ISO: Geneva, Switzerland, 2005.

6. International Standardization for Standardization. ISO 11146-3, Lasers and Laser-Related Equipment-Test Methods for Laser Beam Widths, Divergence Angles and Beam Propagation Ratios-Part 3: Intrinsic and Geometrical Laser Beam Classification, Propagation and Details of Test Methods; ISO: Geneva, Switzerland, 2004.

7. Bouafia, M.; Bencheikh, H.; Bouamama, L.; Weber, H. M² quality factor as a key to mastering laser beam propagation. Proc. SPIE 2004, 5456, 130-140.

8. Paschotta, R. Beam quality deterioration of lasers caused by intracavity beam distortions. Opt. Express 2006, 14, 6069-6074. [CrossRef] [PubMed]

9. Feng, G.-Y.; Zhou, S. Discussion of comprehensive evaluation on laser beam quality. Chin. J. Laser 2009, 36, 1643-1653.

10. Newburgh, G.A.; Michael, A.; Dubinskii, M. Composite Yb:YAG/SiC-prism thin disk laser. Opt. Express 2010, 18, 17066-17074. [CrossRef] [PubMed]

11. Borgentun, C.; Bengtsson, J.; Larsson, A. Full characterization of a high-power semiconductor disk laser beam with simultaneous capture of optimally sized focus and far-field. Appl. Opt. 2011, 50, 1640-1649. [CrossRef] [PubMed]

12. Xiang, Z.; Wang, D.; Pan, S.; Dong, Y.; Zhao, Z.; Li, T.; Ge, J.; Liu, C.; Chen, J. Beam quality improvement by gain guiding effect in end-pumped $\mathrm{Nd}: \mathrm{YVO}_{4}$ laser amplifiers. Opt. Express 2011, 19, 21060-21073. [CrossRef] [PubMed]

13. Scaggs, M.; Haas, G. Real time monitoring of thermal lensing of a multikilowatt fiber laser optical system. In Proceedings of the Laser Resonators, Microresonators, and Beam Control XIV, San Francisco, CA, USA, 22-25 January 2012.

14. Gong, M.L.; Qiu, Y.; Huang, L.; Liu, Q.; Yan, P.; Zhang, H.T. Beam quality improvement by joint compensation of amplitude and phase. Opt. Lett. 2013, 38, 1101-1103. [CrossRef] [PubMed] 
15. Schäfer, B.; Mann, K. Determination of beam parameters and coherence properties of laser radiation by use of an extended Hartmann-Shack wave-front sensor. Appl. Opt. 2002, 41, 2809-2817. [CrossRef] [PubMed]

16. Schäfer, B.; Lübbecke, M.; Mann, K. Hartmann-Shack wave front measurements for real time determination of laser beam propagation parameters. Rev. Sci. Instrum. 2006, 77, 053103. [CrossRef]

17. Neubert, B.J.; Huber, G.; Scharfe, W. On the problem of $\mathrm{M}^{2}$ analysis using Shack-Hartmann measurements. J. Phys. D Appl. Phys. 2001, 34, 2414-2419. [CrossRef]

18. Sheldakova, J.V.; Kudryashov, A.V.; Zavalova, V.Y.; Cherezova, T.Y. Beam quality measurements with Shack-Hartmann wavefront sensor and $\mathrm{M}^{2}$-sensor: Comparison of two methods. In Proceedings of the Laser Resonators and Beam Control IX, San Jose, CA, USA, 22-24 January 2007.

19. Lambert, R.W.; Cortés-Martínez, R.; Waddle, A.J.; Shephard, J.D.; Taghizadeh, M.R.; Greenaway, A.H.; Hand, D.P. Compact optical system for pulse-to-pulse laser beam quality measurement and applications in laser machining. Appl. Opt. 2004, 43, 5037-5046. [CrossRef] [PubMed]

20. Schmidt, O.A.; Schulze, C.; Flamm, D.; Brüning, R.; Kaiser, T.; Schröoter, S.; Duparré, M. Real-time determination of laser beam quality by modal decomposition. Opt. Express 2011, 19, 6741-6748. [CrossRef] [PubMed]

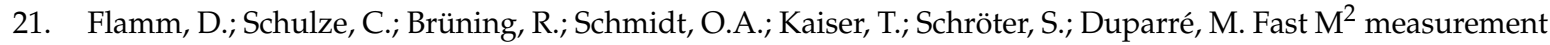
for fiber beams based on modal analysis. Appl. Opt. 2012, 51, 987-996. [CrossRef] [PubMed]

22. Schulze, C.; Flamm, D.; Duparré, M.; Forbes, A. Beam-quality measurements using a spatial light modulator. Opt. Lett. 2012, 37, 4867-4869. [CrossRef]

23. Offerhaus, H.L.; Edwards, C.B.; Witteman, W.J. Single shot beam quality $\left(\mathrm{M}^{2}\right)$ measurement using a spatial Fourier transform of the near field. Opt. Commun. 1998, 151, 65-68. [CrossRef]

24. Du, Y.-Z.; Feng, G.-Y.; Li, H.; Cai, Z.; Zhao, H.; Zhou, S. Real-time determination of beam propagation factor by Mach-Zehnder point diffraction interferometer. Opt. Commun. 2013, 287, 1-5. [CrossRef]

25. Feldman, M.; Mockler, D.J.; English, R.E.; Byrd, J.L., Jr.; Salmon, J.T. Self-referencing Mach-Zehnder interferometer as a laser system diagnostic. In Proceedings of the Active and Adaptive Optical Systems, San Diego, CA, USA, 22-24 July 1991.

26. Rhoadarmer, T.A. Development of a self-referencing interferometer wavefront sensor. Proc. SPIE Adv. Wavefront Control Methods Devices Appl. II 2004, 5553, 112-126.

27. Nemes, G.; Siegman, A.E. Measurement of all ten second-order moments of an astigmatic beam by the use of rotating simple astigmatic (anamorphic) optics. J. Opt. Soc. Am. A 1994, 11, 2257-2263. [CrossRef]

28. Kochkina, E.; Wanner, G.; Schmelzer, D.; Tröbs, M.; Heinzel, G. Modeling of the general astigmatic Gaussian beam and its propagation through 3D optical systems. Appl. Opt. 2013, 52, 6030-6040. [CrossRef] [PubMed]

29. Deng, G.; Feng, G.; Li, W.; Zhang, T.; Liao, H.; Zhou, S. Experimental study of beam quality factor $\mathrm{M}^{2}$ matrix for non-circular symmetry beam. Chin. J. Laser 2009, 36, 2014-2018. [CrossRef]

30. Li, W.; Feng, G.; Huang, Y.; Deng, G.; Huang, Y.; Chen, J.; Zhou, S. Matrix formulation of the beam quality of the Hermite-Gaussian beam. Laser Phys. 2009, 19, 1-6. [CrossRef]

31. Li, W.; Feng, G.-Y.; Huang, Y.; Li, G.; Yang, H.-M.; Xie, X.-D.; Chen, J.-G.; Zhou, S.-H. M² factor matrix for two-dimensional Hermite-Gaussian beam. Acta Phys. Sin. 2009, 58, 2461-2466.

32. Liu, X.-L.; Feng, G.-Y.; Li, W.; Tang, C.; Zhou, S.-H. Theoretical and experimental study on $\mathrm{M}^{2}$ factor matrix for astigmatic elliptical Gaussian beam. Acta Phys. Sin. 2013, 62, 194202.

33. Takeda, M.; Ina, H.; Kobayashi, S. Fourier transform method of fringe pattern analysis for computer-based topography and interferometry. J. Opt. Soc. Am. 1982, 72, 156-160. [CrossRef]

34. Kohler, D.R.; Gamiz, V.L. Interferogram reduction for radial-shear and local-reference-holographic interferograms. App. Opt. 1986, 25, 1650-1652. [CrossRef]

35. Notaras, J.; Paterson, C. Demonstration of closed-loop adaptive optics with a point-diffraction interferometer in strong scintillation with optical vortices. Opt. Express 2007, 15, 13745-13756.

36. Notaras, J.; Paterson, C. Point-diffraction interferometer for atmospheric adaptive optics in strong scintillation. Opt. Commun. 2008, 281, 360-367. [CrossRef]

37. Goodman, J.W. Introduction to Fourier Optics, 2nd ed.; McGraw-Hill: New York, NY, USA, 1968; pp. 63-90.

38. Smartt, R.N.; Stell, W.H. Theory and Application of Point-Diffraction Interferometers. Jpn. J. Appl. Phys. 1975, 14, 351-356. [CrossRef]

39. Koliopoulos, C.; Kwon, O.; Shagam, R.; Wyant, J.C.; Hayslett, C.R. Infrared point-diffraction interferometer. Opt. Lett. 1978, 3, 118-120. [CrossRef] [PubMed] 
40. Mercer, C.R.; Creath, K. Liquid-crystal point-diffraction interferometer for wave-front measurement. Appl. Opt. 1996, 35, 1633-1642. [CrossRef] [PubMed]

41. Lago, E.L.; dela Fuente, R. Amplitude and phase reconstruction by radial shearing interferometry. Appl. Opt. 2008, 47, 372-377. [CrossRef]

42. Bone, D.J.; Bachor, H.-A.; Sandeman, R.J. Fringe-pattern analysis using a 2-D Fourier transform. Appl. Opt. 1986, 25, 1653-1660. [CrossRef] [PubMed]

43. Roddier, C.; Roddier, F. Interferogram analysis using Fourier transform techniques. Appl. Opt. 1987, 26, 1668-1673. [CrossRef] [PubMed]

44. Mendlovic, D.; Zalevsky, Z.; Konforti, N. Computation considefactorns and fast algorithms for calculating the diffraction integral. J. Mod. Opt. 1997, 44, 407-414. [CrossRef]

45. Kogelnik, H.; Li, T. Laser beams and resonators. Appl. Opt. 1996, 5, 1550-1567. [CrossRef] [PubMed]

46. Siegman, A.E. Lasers; University Science Books: Mill Valley, CA, USA, 1986; pp. 777-811.

(C) 2016 by the author; licensee MDPI, Basel, Switzerland. This article is an open access article distributed under the terms and conditions of the Creative Commons Attribution (CC-BY) license (http://creativecommons.org/licenses/by/4.0/). 